BENTHAM OPEN
CrossMark
Content list available at: www.benthamopen.com/TOCIEJ/
DOI: $10.2174 / 1874149501812010187$

RESEARCH ARTICLE

\title{
Physical Characterization of Alterites for the Manufacture of Compressed Earth Blocks
}

\author{
Kamga Djoumen Tatiana ${ }^{1}$, Codjo Luc Zinsou ${ }^{2}$, Vouffo Marcel $^{3}$ and Ngapgue François ${ }^{1, *}$ \\ ${ }^{I}$ FOTSO Victor University Institute of Technology, University of Dschang, Dschang, Cameroon \\ ${ }^{2}$ Abomey-Calavi Avanced School of Engineering, Abomey-Calavi, Benin \\ ${ }^{3}$ Faculty of Science, University of Dschang, Dschang, Cameroon
}

Received: March 20, 2018

Revised: April 16, 2018

Accepted: June 10, 2018

\section{Abstract:}

\section{Introduction:}

In the present work, the physical characteristics of two alterites $\left(\mathrm{S}_{1}\right.$ and $\left.\mathrm{S}_{2}\right)$ used for Compressed Earth Blocks (CEB) manufacture were studied. The results obtained have shown that $\mathrm{S}_{1}$ and $\mathrm{S}_{2}$ consist of inorganic clays.

\section{Methods:}

The material $S_{1}$ is a plastic soil of very soft consistency and $S_{2}$ is a low plastic soil of very soft consistency. It was shown that the natural alterites studied are not suitable for the CEB manufacture. In order to improve the granulometry of these materials, a physical correction by adding sand in various proportions were proposed. With the aim of verifying the validity of the elaborated proposals, samples of CEB manufactured from materials stabilized with sand were manufactured and tested in the laboratory.

\section{Results and Conclusion:}

The results obtained show that, concerning the $S_{1}$ material, the tensile strength is satisfactory for the sand/soil ratios of $1 / 3,1 / 2$ and $2 / 3$. The abrasion resistance and the water absorption coefficient are satisfactory for the ratios of $1 / 3$ and $1 / 2$, respectively. For all the sand/soil ratios, the compressive strength has remained lower than that required for CEB as materials for load-bearing walls. For the $\mathrm{S}_{2}$ material, all the sand/soil ratios enable the improvement of the CEB characteristics, but these still below the required values. From all the foregoing, it follows that the studied alterites, improved by the addition of sand, can be used for the manufacture of compressed earth blocks to be used for the construction of non-load bearing walls.

Keywords: Compressed earth blocks, Alterites, Characterization, Stabilization, Strength, Material $\mathrm{S}_{1}$.

\section{INTRODUCTION}

Due to their high cost, modern materials are almost inaccessible for the greater part of the population of developing countries. Due to the problems that these countries come across, it is imperative to promote local materials adapted relating the cost to the quality. Among the constructions in local materials, is the construction in earth material. According to several authors, construction in earth material has many advantages which are: low cost of construction [1], aesthetics, thermal [2] and acoustic isolation, the low maintenance cost, high resistance to fire, flexibility in its application, and several surface textures can be realized, which produces a more acceptable architecture and assure the preservation of the cultural patrimony. It is estimated that $30 \%$ of earthen houses in the world are in Africa, concentrated in countries said to be poor. In Cameroon, approximately $70 \%$ of houses, both in the rural and urban zones are made in lateritic earth blocks [3]. However, the number of scientific work done on alterites remains relatively low.

\footnotetext{
* Address correspondence to this author at the FOTSO Victor University Institute of Technology, University of Dschang, Dschang,
} Cameroon; Tel: +237-699547394; E-mail: fgapguefcr@gmail.com 
P'kala A [1]. carried out the characterization of CEB in simple compression, with application to masonry. Sivwira Malukite R [4]. has shown the influence of water-repellent admixtures on the physico-mechanical properties of CEB made from South Kivu alterites. Fopossi J. A [5]. has shown the effects of stabilizers on the physical and mechanical properties of clay, using Mangu (Kenya) soils. Moimo Ndanga R. A [6]. has studied the physical characteristics of Toutsang (Dschang) alterites for the production of compressed earth blocks.

None of these works has studied the characteristics of sand-mixed alterites for the manufacture of CEB. Taking into consideration the high solicitation of the earth materials in construction, it is essential to know their physical characteristics and to find out stabilization models favorable to the resistance of the habitat.

The objective of the present work is to study the physical characteristics of two alterites and to elaborate propositions in view of their amelioration for the production of compressed earth blocks. Indeed, faced with the high costs of building materials, the new challenges of the construction industry is the need to produce low-cost sustainable buildings accessible to all strata of the population and to develop the use of materials sustainable, locally available, and low environmental impact, such as CEB. Everywhere in the worldwide, natural earth is abundantly available; most of the people are limited to precarious revenue and the higher costs of construction with imported materials. CEB offers cultural and traditional heritage to populations. While the cement has better cohesive properties of materials by hydration, the properties of sand is to reduce plastic shrinkage. This justifies the reason to focus this research on improving the properties of strength and durability by studying the effect of diverse combinations of soil, sand and cement on the CEB in order to upgrade the construction of buildings. Sustainable solutions should benefit to all social strata.

\section{METHODS}

Experimental studies were done on earth samples collected from the Maka'a quarry in Foreke-Dschang, Cameroon. The literature search provided insight into previous work on alterites and their use in the manufacture of CEB.

The touch/smell/washing tests have allowed an apparent classification of the material and its organic matter content. The bottle tests have permitted us to verify the presence of all the elements (sand, silts, clay). The observation of the soil cohesion, the proportion and the quality of clay particles have been carried out using the cigar and the bursting tests. All these tests have been performed according to CRATerre-EAG and ENTPE recommendations [7].

The water content of the material was determined by drying in an oven according to NF P94-050 [8]. The absolute density was determined using the pycnometer method according to the NF P 94-054 norm [9]. The dry density $\left(d_{d}\right)$, the porosity $(n)$, the void ratio (e) and the saturation ratio $\left(S_{r}\right)$ of the studied samples were determined by the use of the relationships existing between state parameters.

The particle size analysis of the materials was carried out by sieving after washing, according to standard NF P 94-056 [10].

The liquid limit has been determined using a cone penetrometer, according to standard NF P 94-052-1 [11]. The plastic limit was determined using the roller method in accordance with standard NF P 94-051 [12]. The results obtained made it possible to determine the indices of plasticity, consistency and liquidity using the relationships existing between the physical characteristics of the materials.

In view of the amelioration of the characteristics of the soil, the physical stabilization was done by adding sand in diverse proportions. With the aim of verifying the validity of the elaborated propositions, samples of CEB were manufactured and tested with respect to compression following the NF EN 14617 -15 [13] standard. Tensile strength and absorption tests were carried out according to the procedures of Center for the Development of the Enterprise (CDE) [7]. Abrasion test was carried out according to the standard NF EN 530 [14].

\section{RESULTS AND INTERPRETATION}

\subsection{Description of Alteration Profiles}

In the quarry producing the studied materials, the presence of an upper humeral horizon, a lower horizon consisting of reddish materials $\left(\mathrm{S}_{1}\right)$ and a medium horizon consisting of purplish red materials $\left(\mathrm{S}_{2}\right)$ were noted. The latter contains a huge amount of limestone. The materials of the last two horizons are the subject of this study because of their abundance, their wide use in construction and the important problems they pose. 


\subsection{Physical Characteristics of the Soil}

The physical characteristics of the studied soils presented in this paragraph are essentially state parameters, particle size distribution and consistency parameters.

\subsubsection{Identification of the Materials}

The identification tests on materials have enabled us to obtain the results presented in Table $\mathbf{1}$.

Table 1. Results obtained from the identification tests.

\begin{tabular}{|c|c|c|c|c|}
\hline Materials & $\begin{array}{l}\text { Touch } / \text { Smell/ } \\
\text { Washing Test }\end{array}$ & Cigar Test & Pellet Test & Bottle Test \\
\hline $\mathbf{S}_{1}$ & $\begin{array}{l}\text { - No smell ; } \\
\text { - Very cohesive }\end{array}$ & Average value of the lengths: $7.13 \mathrm{~cm}$ & $\begin{array}{c}\text { - High shrinkage; } \\
\text { - Earth crashes with difficulty between the } \\
\text { fingers }\end{array}$ & Presence of all the elements \\
\hline $\mathbf{S}_{2}$ & $\begin{array}{c}\text { - No smell } \\
\text { - Less cohesive } \\
\text {-Abrasive sensation. }\end{array}$ & Average value of the lengths: $5.46 \mathrm{~cm}$ & $\begin{array}{l}\text { - Low shrinkage } \\
\text { - Earth crashes easily between the fingers }\end{array}$ & Absence of all the elements \\
\hline
\end{tabular}

Table 1 shows that the material $S_{1}$ is very cohesive, presents a great shrinkage and is not easily ground in between fingers, due to the fact that the material is rich in clay particles. On the other hand, the material $\mathrm{S}_{2}$ is less cohesive, presents a low shrinkage and is easily ground in between fingers, due to the fact that the material is poor in clay particles. The cigar test gives average values of $7.13 \mathrm{~cm}$ and $5.46 \mathrm{~cm}$, respectively for the materials $S_{1}$ and $S_{2}$. These values, found in the range of 5 and $15 \mathrm{~cm}$ indicate that these materials are a good soil for the manufacturing of CEB, despite the fact that there is an absence of certain elements in the material $\mathrm{S}_{2}$.

\subsubsection{State Parameters of Studied Materials}

The values of the water content, the absolute density, the dry density, the porosity, the void ratio and the degree of saturation of the soils studied are presented in Table 2 .

Table 2. State parameters of natural materials.

\begin{tabular}{|c|c|c|c|c|c|c|}
\hline Material & Water Content (\%) & Absolute Density & Dry Density & Porosity (\%) & Void Ratio & Saturation Ratio (\%) \\
\hline $\mathbf{S}_{\mathbf{1}}$ & 23.98 & 2.53 & 1.29 & 48 & 0.96 & 63 \\
\hline $\mathbf{S}_{\mathbf{2}}$ & 25.31 & 2.50 & 1.26 & 49 & 0.98 & 65 \\
\hline
\end{tabular}

Table 2 shows that the material $\mathrm{S}_{1}$ has a water content of $23.98 \%$ at the moment of sampling and a saturation ratio of $63 \%$. Its absolute density and dry density are 2.53 and 1.29 respectively. The values of the void ratio and the porosity are 0.96 and $48 \%$ respectively. The water content and saturation ratio of the material $\mathrm{S}_{2}$ are $25.31 \%$ and $65 \%$ respectively. The absolute density, the dry density, the void ratio and the porosity are $2.50,1.26,0.98$ and $48 \%$, respectively.

The high value of water content shows that the studied materials are sensitive to water. This can be explained by the high clay particles content of the materials. The values of the density parameters are very close together. This is explained by the fact that these materials are obtained from the same mother rock, which have been formed at the same conditions and are of the same degree of alteration. This shows that they are homogenous.

\subsubsection{Particle Size Parameters of Studied Materials}

The particle size distribution of the studied materials is presented in Fig. (1).

Fig. (1) shows that the dimensions of the coarse grains are $20 \mathrm{~mm}$ and $5 \mathrm{~mm}$, respectively for $\mathrm{S}_{1}$ and $\mathrm{S}_{2}$ materials. The weight distribution of grains of the different studied materials is as follows:

$-16.28 \%$ of gravel, $33.99 \%$ coarse sand, $6.49 \%$ of fillers sands and $43.24 \%$ fines for the reddish material $\left(\mathrm{S}_{1}\right)$;

$-1.45 \%$ gravel, $36.9 \%$ coarse sand, $4.52 \%$ fillers sand and $57.13 \%$ fines for the red purplish material $\left(\mathrm{S}_{2}\right)$.

The particle size distribution curves on Fig. (1) shows that the studied materials are rich in fine particles, which certify a high degree of chemical weathering. In addition, the curves look to be the same and are close to each other, 
which shows that these materials come from the same rock and are of the same degree of weathering. The influence of this particle size distribution on the consistency characteristics of the studied materials is considerable.

He shows that

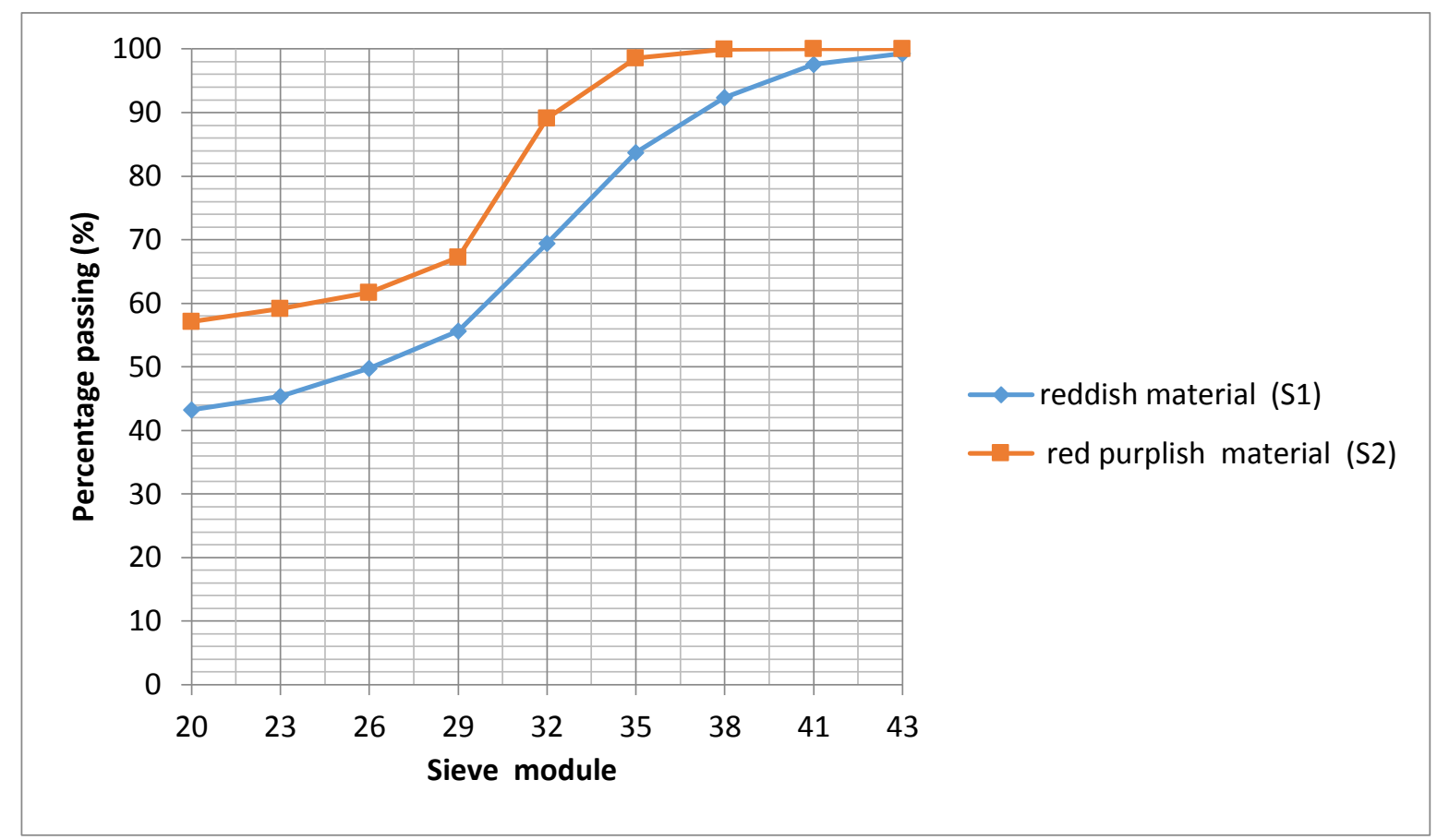

Fig. (1). particle size distribution curves of the $S_{1}$ and $S_{2}$ materials.

\subsubsection{Consistency Parameters}

The liquid limit $\left(\mathrm{W}_{\mathrm{L}}\right)$, the plastic limit $\left(\mathrm{W}_{\mathrm{P}}\right)$, the plasticity index $(\mathrm{Ip})$, the liquidity index $\left(\mathrm{I}_{\mathrm{L}}\right)$ and the consistency index (Ic) obtained are presented in Table 3.

Table 3. Consistency characteristics of the studied materials.

\begin{tabular}{|c|c|c|c|c|c|}
\hline Materials & $\mathbf{W}_{\mathbf{L}} \mathbf{( \% )}$ & $\mathbf{W}_{\mathbf{P}} \mathbf{( \% )}$ & $\mathbf{I p} \mathbf{( \% )}$ & $\mathbf{I}_{\mathbf{L}} \mathbf{( \% )}$ & $\mathbf{I}_{\mathbf{c}}(\mathbf{\%})$ \\
\hline $\mathbf{S}_{\mathbf{1}}$ & 60.60 & 43.77 & 16.83 & -1.17 & 2.17 \\
\hline $\mathbf{S}_{\mathbf{2}}$ & 63.10 & 56.07 & 7.03 & -4.38 & 5.38 \\
\hline
\end{tabular}

Table 3 shows that $\mathrm{W}_{\mathrm{L}}$ and $\mathrm{W}_{\mathrm{p}}$ of the reddish material $\left(\mathrm{S}_{1}\right)$ are respectively $60.60 \%$ and $43.77 \%$. Its plasticity index is $16.83 \%$. The liquidity and consistency indices are respectively -1.17 and $2.17 \%$. These values show that the material $\mathrm{S}_{1}$ is a plastic soil found in the solid or semi-solid state, having a very soft consistency. Concerning the red purplish $\left(\mathrm{S}_{2}\right)$, $\mathrm{W}_{\mathrm{L}}, \mathrm{W}_{\mathrm{p}}, \mathrm{I}_{\mathrm{p}}, \mathrm{I}_{\mathrm{L}}$, and $\mathrm{I}_{\mathrm{c}}$ are $63.1 \%, 56.07 \%, 7.03 \%,-4.38$ and $5.38 \%$, respectively. These values show that the material $\mathrm{S}_{2}$ is a little plastic soil found in the solid or semi-solid state and having a very soft consistency.

According to the Casagrande plasticity abacus [15], the values of the plasticity index show that the materials $S_{1}$ and $\mathrm{S}_{2}$ consist of inorganic clays. The absence of organic matter in these clays shows that these materials do not present any plant contamination. According to the soil classification scale, the material $\mathrm{S}_{1}$ is a plastic soil of very soft consistency and $S_{2}$ is a low plastic soil of very soft consistency. The position of the points representing the materials studied in the plasticity diagram is presented in Fig. (2).

Fig. (2) shows that the points representing the materials studied do not fit into the preferred plasticity zone recommended by standards [15] for the manufacture of CEB. This is due to the high content of fine particles of these materials. It follows that it is necessary to correct the particle size composition of the materials studied before their use in the manufacture of CEB. 


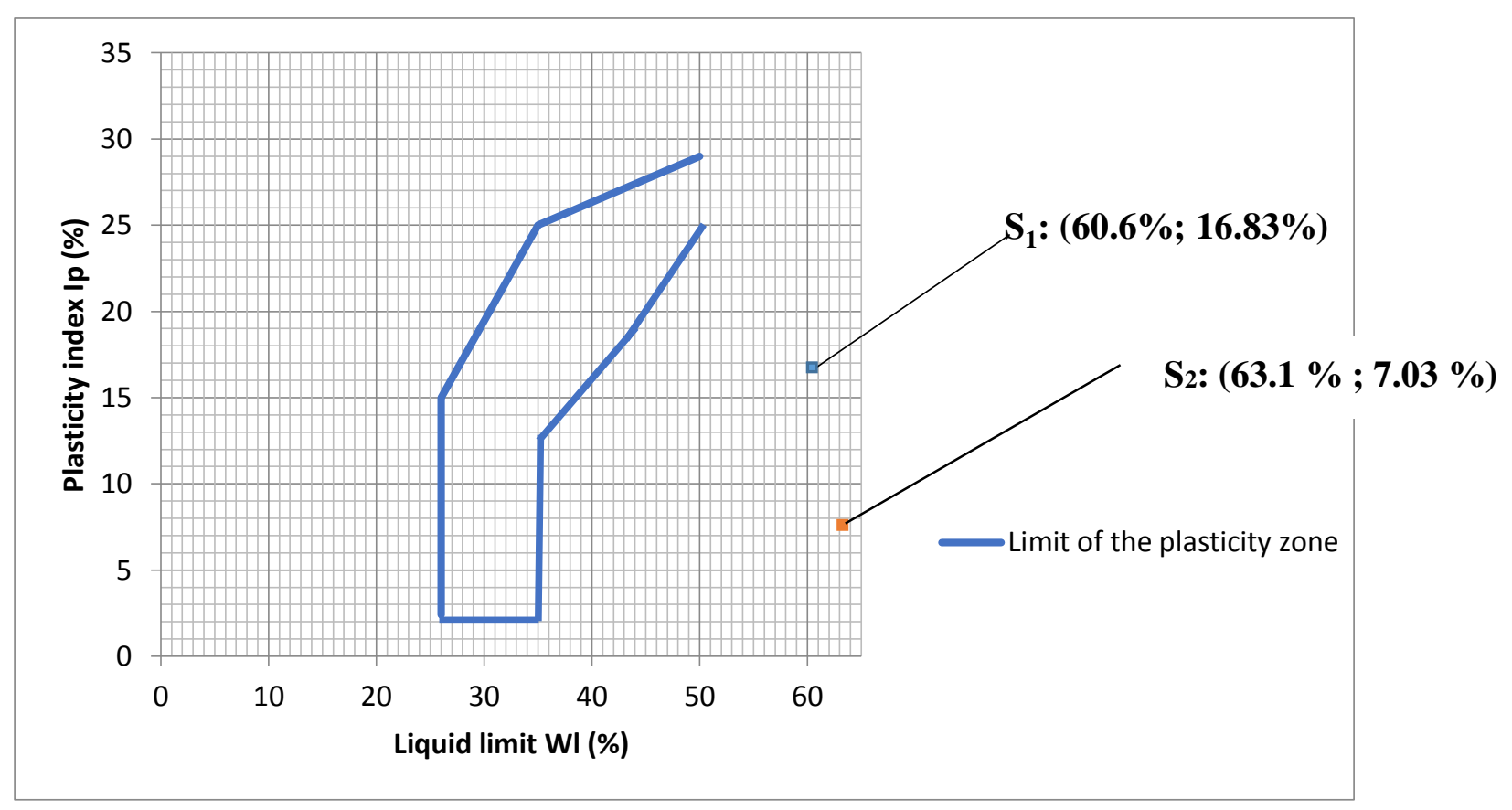

Fig. (2). Position of the materials $S_{1}$ and $S_{2}$ in the plasticity diagram.

\section{PROPOSALS IN VIEW OF QUALITY PRODUCTION OF CEB FROM THE STUDIED MATIALS}

\subsection{PROPOSALS IN VIEW OF QUALITY PRODUCTION OF CEB}

In the present work, physical stabilization by adding sand to natural materials was proposed. To this effect, sand was collected from Fombap quarry which is a good sand for such stabilization according to its characteristics [16 - 18]. The proportions of sand used for the physical stabilization were $1 / 2,1 / 3$ and $2 / 3$ of the soil volume.

\subsection{VERIFICATION OF THE VALIDITY OF ELABORATED PROPOSALS}

With the aim of verifying the validity of the elaborated proposals, CEB samples were made:

- CEB samples with no ameliorated materials (natural materials);

- CEB samples stabilized by the addition of sand (physical stabilization) at diverse proportions;

- CEB samples stabilized by the addition of sand and Portland at diverse proportions. All the CEB samples were submitted to compressive, tensile, abrasion and absorption tests. The results obtained for stabilized CEB and CEB after physical stabilization are presented in the Tables $\mathbf{4}$ and $\mathbf{5}$.

According to Table 4, the values of the dry compressive strength of CEB produced from the reddish and red purplish materials, respectively of $1.90 \mathrm{MPa}$ and $1.69 \mathrm{MPa}$ are lower than the minimum (4 MPa) required by the norms. The dry tensile, strength of $0.42 \mathrm{MPa}$ and $0.37 \mathrm{MPa}$ obtained respectively for the reddish and red purplish are lower than the minimum value of $0.5 \mathrm{MPa}$, required by the standard. Concerning the abrasion strength, the rate of mass loss by abrasion is $3.36 \%$ and $8.90 \%$, respectively for materials $\mathrm{S}_{1}$ and $\mathrm{S}_{2}$. If the first value is admissible because it is lower than the ultimate value of $5 \%$, then the second is not. The values obtained showed that CEB produced from the studied natural materials cannot sufficiently resist to diverse mechanical solicitations.

Table 4. Results of mechanical tests and absorption rate of water in the CEB with the primary basic natural material.

\begin{tabular}{|c|c|c|c|c|}
\hline Materials Used & Dry Compressive Strength (MPa) & $\begin{array}{c}\text { Dry Tensile Strength } \\
\text { (MPa) }\end{array}$ & Abrasion Coefficient (\%) & $\begin{array}{c}\text { Water Absorption by Capillarity } \\
\text { (\%) }\end{array}$ \\
\hline $\mathbf{S}_{\mathbf{1}}$ & 1.90 & 1.69 & 3.36 & 17.14 \\
\hline $\mathbf{S}_{\mathbf{2}}$ & 0.42 & 0.37 & 8.90 & 23.37 \\
\hline
\end{tabular}


Table 5. Results obtained from the mechanical tests and the absorption rates of water after physical stabilization of CEB.

\begin{tabular}{|c|c|c|c|c|c|}
\hline \multicolumn{2}{|c|}{ Materials Used } & $\begin{array}{c}\text { Dry Compressive Strength } \\
\text { (MPa) }\end{array}$ & $\begin{array}{c}\text { Dry Tensile Strength } \\
\text { (MPa) }\end{array}$ & Abrasion Coefficient (\%) & $\begin{array}{c}\text { Water Absorption by Capillarity } \\
\text { (\%) }\end{array}$ \\
\hline Soils & Sand/Soil ratios & 1.92 & 1.67 & 3.85 & 18.84 \\
\hline \multirow{3}{*}{$\mathbf{S}_{1}$} & $1 / 3$ & 2.1 & 1.85 & 8.37 & 10.38 \\
\cline { 2 - 6 } & $1 / 2$ & 2.01 & 1.73 & 10.32 & 23.1 \\
\hline \multirow{3}{*}{$\mathbf{S}_{2}$} & $2 / 3$ & 0.3 & 0.27 & 25.36 & 25.75 \\
\cline { 2 - 6 } & $1 / 3$ & 0.44 & 0.39 & 24.62 & 22.01 \\
\cline { 2 - 6 } & $1 / 2$ & 0.4 & 0.35 & 23.83 & 37.37 \\
\hline
\end{tabular}

The rate of capillary absorption of CEB is in the range $17.14 \%$ and $23.37 \%$ respectively for the materials $S_{1}$ and $S_{2}$. These values are higher than the allowable absorption rate which is $10 \%$ according to the norms. Thus, masonries in CEB from the natural materials studied are not convenient in a capillary environment.

Table 5 shows the dry compressive and tensile strength of CEBS made from the reddish materials are $1.92 \mathrm{MPa}$ and 1.67 MPa, 2.1 MPa and 1.85MPa, 2.01MPa and 1.73 MPa, respectively for sand/soil ratio of $1 / 3 ; 1 / 2$ and $2 / 3$. For CEBS manufactured from the red purplish material, the dry compressive and tensile strength are $0.3 \mathrm{MPa}$ and 0.27 $\mathrm{MPa}, 0.44 \mathrm{MPa}$ and $0.39 \mathrm{MPa}, 0.4$ and $0.35 \mathrm{MPa}$, respectively for sand/soil ratio of $1 / 3 ; 1 / 2$ and $2 / 3$. The values of dry compressive strength are less than the minimum allowable value of $4 \mathrm{MPa}$. Concerning dry tensile strength, the results obtained are satisfactory with the material $S_{1}$ and rather poor for the material $S_{2}$ because the values obtained are less than the minimum value of $1 \mathrm{MPa}$ for CEB.

Concerning the abrasion strength, the values of mass loss by abrasion are $3.85 \%, 8.37 \%$ and $10,32 \%$, respectively for the stabilization with $1 / 3,1 / 2$ and $2 / 3$ sand/soil ratio for the $S_{1}$ material For the $S_{2}$ material, these values are $25.36 \%$, $24.62 \%$ and $23.83 \%$ for the sand/soil ratio of $1 / 3,1 / 2$ and $2 / 3$, respectively. We note that for the stabilization with sand/soil ratio of $1 / 3$, the $S_{1}$ material resists to abrasion. For $1 / 2$ and $2 / 3$ ratio, this material does not resist to abrasion since the values remain higher than the allowable value of 5\% [6]. As for the material $\mathrm{S}_{2}$, there is no physical stabilization which gives satisfaction.

For material $\mathrm{S}_{1}$, the rates of capillary absorption of the CEB are $18.84 \%, 10.38 \%$ and $23.21 \%$, respectively for $1 / 3$, $1 / 2$ and $2 / 3 \mathrm{sand} /$ Soil ratio. These values are respectively $25.75 \%, 22.01 \%$ and $37.37 \%$, for the material $\mathrm{S}_{2}$. They are always very high with the exception of material $S_{1}$ in the proportion $1 / 2$ which is near to the absorption rate admissible by the norms $(10 \%)$.

It springs out from this study that proportion $1 / 2 \mathrm{sand} /$ soil ratio is the best with regards to the mechanical strength obtained. Meanwhile, this mechanical strength are still very low as compared to the prescribed minimum limit values, principally for the material $\mathrm{S}_{2}$. The stabilization by the addition of cement (chemical stabilization) is known to be necessary to obtain good quality blocks.

\section{CONCLUSION}

The present work aimed to study the physical characteristics of alterites and proposing improving measures for the production of compressed earth blocks. To reach this goal, the identification tests for the natural primary materials were carried out. A comparison of the mechanical characteristics of compressed earth blocks (CEB) manufactured from the natural primary materials to those of CEB produced from the material having undergone stabilization by adding sand at different proportions was done. The obtained results showed that:

- According to HRB classification, reddish materials and red purplish material are respectively from class A-7-5(20) and A-5(12);

- The fine particles content of natural materials is very high, which do not allow them to be used for making CEB.

In order to improve the granulometry of these alterites, a physical correction by adding sand in various proportions was proposed. With the aim of verifying the validity of the elaborated proposals, samples of CEB manufactured from stabilized alterites were manufactured and tested in the laboratory. The results obtained showed that, for material S1, the tensile strength is satisfactory for sand / soil ratios of $1 / 3,1 / 2$ and $2 / 3$. The abrasion resistance and the water absorption coefficient are satisfactory respectively for the ratios of $1 / 3$ and $1 / 2$. With regards to compressive strength, 
all sand / soil ratios used gave values below those allowed. For the $\mathrm{S}_{2}$ material, all sand / soil ratios have also improved the CEB characteristics, but these are still below the required values. From all the foregoing, it follows that the studied alterites, improved by the addition of sand, can be used for the manufacture of compressed earth blocks to be used for the construction of non-load bearing walls. The compressed earth blocks made from unstabilized materials can be used for filling the walls of reinforced concrete frame buildings, provided that the surface of the walls is protected with cement stabilized clay.

\section{CONSENT FOR PUBLICATION}

Not applicable.

\section{CONFLICT OF INTEREST}

The authors declare no conflict of interest, financial or otherwise.

\section{ACKNOWLEDGEMENTS}

The work presented in this paper was carried out at the FOTSO Victor University Institute of Technology Civil Engineering Laboratory (University of Dschang, Cameroon). The support of the Director of the said University Institute is acknowledged for allowing all the tests to be carried out free of charge in the Institution Civil Engineering Laboratory.

\section{REFERENCES}

[1] A. P'kala, "Characterization in simple compression of compressed earth blocks (BTC): Application to the masts (BTC-Mortar)", Thèse de PhD, Institut National des Sciences Appliquées de Lyon, France, 2000.

[2] P. Meukam, A. Noumowe, Y. Jannot, and R. Duval, "Thermophysical and mechanical characterization of stabilized earth bricks for thermal insulation of buildings", Materials and structures/Materials and constructions, RILEM, vol. 36, no. 261, pp. 453-460, 2003.

[3] L. Mbumbia, A.M. de Wilmars, and J. Tirlocq, "Performance characteristics of lateritic soil bricks fired at low temperature: A case study of Cameroon", Constr. Build. Mater., vol. 14, no. 3, pp. 121-131, 2000. [http://dx.doi.org/10.1016/S0950-0618(00)00024-6]

[4] R.S. Malukite, "Influence of a water-repellent admixture on the physico-mechanical properties of Portland cement stabilized CEB", M.E. thesis, ULPGL - Goma, R.D. Congo, 2015.

[5] J.A. Fopossi, "Effects of stabilizers on the physical and mechanical properties of clay blocks: A case study of Mangu soils, Kenya", M.S. thesis, Panafrican University Institute for Basic Sciences, Technology and Innovation: Nairobi, Kenya, 2014.

[6] R.A.M. Ndanga, "Physical characterization of Toutsang alterites (Dschang) for the production of compressed earth blocks", M.E. thesis, N.A.S.E., University of Yaounde I: Cameroon, 2012.

[7] "Compressed earth blocks: Testing Procedures", Technology Series, CDE, no. 16, Belgium, 2000.

[8] AFNOR, NF P 94-056, "Soils: recognition and testing. Determination of the water content of the materials. Oven method", Paris, France, 1995.

[9] AFNOR, NF P 94-054, "Soils: recognition and testing. Determination of the absolute density of soil. Pycnometer method", Paris, France, 1991.

[10] AFNOR, NF P 94-056, "Soils: recognition and testing. Particle size analysis. Method by dry sieving after washing", Paris, France, 1996.

[11] AFNOR, NF P 94-052-1, "Soils: recognition and testing. Determination of Atterberg limits. Part 1: Liquid limit. Penetration cone method", France, Paris, 1995.

[12] AFNOR, NF P 94-051, "Soils: recognition and testing. Determination of the Atterberg limits: Liquid limit with the use of the cup - plastic limit by the roll method", Paris, France, 1993.

[13] AFNOR, NF EN 14617-15, "Agglomerated stone - Test methods - Part 15: Determination of compressive strength", Paris, France, 2005.

[14] AFNOR, NF EN 530, "Abrasion resistance of the material constituting a protective clothing- Test methods", Paris, France, 2010.

[15] V. Robitaille, and D. Tremblay, "Soil mechanics: theory and practice", Modulo, Quebec, Canada, 1997.

[16] AFNOR, NF XP P 13-901, "Compressed earth blocks for walls and partitions", Paris, France, 2001.

[17] T.D. Kamga, "Physical Characterization of Santchou Sands for Best Use in concrete manufacturing", Master of Engineering Thesis, CUINASE, Douala, Cameroon, 2015. 
[18] T.D. Kamga, C.B. Bishweka, G. Kamdjo, and F. Ngapgue, "Physical Characterization of River Sands for their Better Use in Concrete manufacturing", International Journal of Innovative and Scientific Research, vol. 25, no. 2, pp. 517-527, 2016.

\section{(C) 2018 Tatiana et al.}

This is an open access article distributed under the terms of the Creative Commons Attribution 4.0 International Public License (CC-BY 4.0), a copy of which is available at: (https://creativecommons.org/licenses/by/4.0/legalcode). This license permits unrestricted use, distribution, and reproduction in any medium, provided the original author and source are credited. 